\title{
Elogio de la razón mundana. Antropología y política en Kant, Nuria Sánchez Madrid
}

\author{
Héctor Pérez Guido
}

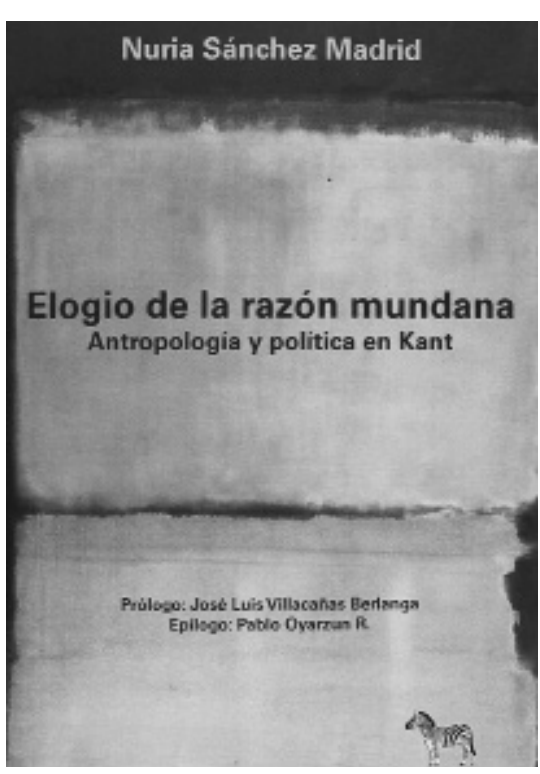

Nuria Sánchez Madrid, Elogio de la razón mundana. Antropología y politica en Kant. Madrid: Ediciones La Cebra, 2018.
Dentro de una corriente que ya puede considerarse una nueva tradición en los estudios kantianos, que pone especial atención en temas que se habían considerado menores, la doctora Nuria Sánchez Madrid, académica e investigadora de la Universidad Complutense de Madrid, entrega en este libro un generoso trabajo de síntesis de una significativa muestra del material publicado respecto a estos temas en lenguas hispana, portuguesa, inglesa, italiana y, por supuesto, alemana.

Como lo reconoce en su introducción, siete nuevas versiones de trabajos antes publicados en revistas, junto con dos nuevos capítulos, se compendian en esta obra abundante en referencias, que recupera a cabalidad su 
propia trayectoria dedicada al estudio del filósofo de Königsberg.

La intención de Sánchez Madrid, como lo evidencia desde el título, es hablar bien sobre aquello que ha sido siempre vilipendiado en Kant por su carácter formal, abstracto y, en cierto sentido, alejado del mundo: la razón mundana; como contraria de la razón pura, adquiere sentido positivo en este libro. Para ello, la autora nos propone una renovada y viva imagen de Kant; en companía de un grupo bastante nutrido de especialistas sobre los cuales, constante y puntualmente, hace mención desde su introducción y en cada apartado del libro.

Los nueve capítulos que componen la totalidad de la obra se encuentran divididos, a su vez, en tres grandes líneas temáticas. La primera de ellas está dedicada al papel de las emociones dentro de la filosofía kantiana. En este apartado, la doctora Sánchez Madrid reconoce la importancia del trabajo de Robert B. Louden en su Kant's Impure Ethics y se adhiere a los estudios de Marcia Baron, Christine Korsgaard, Nancy Sherman, Alix Cohen, Patrick Frierson y Angelica Nuzzo.
El primer ensayo que compone esta sección, tras presentar las principales propuestas de los autores mencionados, nos introduce a las emociones que se comunican con la razón en el pensamiento de Kant, a partir del concepto de Bedürfnis, cuya difícil traducción es "menesterosidad" y nos habla de una "necesidad subjetiva". Es la base de la actividad racional que la motiva en algunos momentos a avanzar, y en otros, a detenerse, impulsada también ante el sentimiento de humillación; se traduce en disposición natural a la metafísica como tarea de especulación.

En el mismo texto, Sánchez Madrid recalca la importancia de la actividad emocional desde el sentimiento de vida como una experiencia que, en el placer y displacer, anticipa una primera manera de orientar el ánimo humano en su elección; sólo de forma secundaria pasa por la conceptualización y la reflexión. En ese mismo sentido, este ensayo hace gala de la filiación kantiana al principio epicureísta del "corazón siempre alegre", como forma de felicidad en la cual los seres humanos tienen que ser educados en sus afectos y emociones. Además, la 
autora expone la relación entre el conocimiento y el sentimiento de placer a partir de la Crítica del juicio; retomando a Leonel Ribeira dos Santos, nos dice que la lógica kantiana estaría basada en la experiencia de un placer originario sobre la relación con la naturaleza desde la captación cognoscitiva, es decir, desde su concordancia.

El segundo ensayo expone cómo el temperamento puede ser visto como suelo de la libertad, esto es, siguiendo lo que Kant desde sus Observaciones sobre el sentimiento de lo bello y lo sublime y la Antropología en sentido pragmático sostuvo acerca de la influencia con que la Naturaleza se hace presente en el ánimo del ser humano. Los cuatro temperamentos corresponden con un orden lógico a cuatro extremos opuestos, y contienen un término medio entre sí. El carácter, obra del artificio humano, viene a reemplazarlos posteriormente, pero el que todos poseamos un temperamento específico nos permite entender que otros también lo tengan y esto nos hace decir, cuando no estamos de acuerdo con ello, que simplemente no sentimos como el otro. El conocimiento de los temperamentos permite, a la vez, que existan maneras de relacionarse entre las distintas personas que los poseen, de ahí su relevancia. De manera semejante, un arte como la fisionómica, orientada a la interpretación de rasgos y gestos en las personas, aproxima a los seres humanos a relacionarse y ejercitarse en el intento de comprender lo que se manifiesta a través de ellos. Para Sánchez Madrid, gran lectora de Blumenberg, esto nos confirma que somos seres semióticos (atravesados por el principium rationis indifferentis), que buscamos significados en todo lo que nos rodea.

En el tercer ensayo, que concluye la primera línea temática del libro, se abordan las pasiones desde las Lecciones de antropología de Kant. Considerando la difícil oposición entre lo práctico y lo patológico, Nuria Sánchez presenta la manera en que las emociones y las pasiones afectan no sólo a la facultad de sentir placer y displacer, sino también a la de desear, desde las reflexiones antropológicas de Kant. La realidad con que las pasiones afectan a la voluntad se hace patente $y$, desde ahí, juega un papel importante en la confección de una teoría moral que no se sujeta al 
normativismo del deber ser. Ligadas al sentimiento de vida, las pasiones también son capaces de dirigir, y hasta de elevar, principios con respecto a sus objetos. Se ve, entonces, que, apartadas de las inclinaciones, las pasiones pueden ser destructivas no por su falta de regulación, sino por la obsesión que pueden producir para alcanzar sus fines. No obstante, pueden ser cultivadas, educadas y dirigidas hacia algún provecho para la humanidad. Son de tal potencia que pueden incurrir en incongruencias como la que Sánchez Madrid advierte respecto al apetito de venganza y el de derecho, en donde este último suplanta al primero, pero ambos tendrían un origen común en el ánimo del hombre. Lo anterior conduce a la autora a comparar la condición humana, desde la visión antropológica de Kant con la metapsicología de Freud, esto es, desde la noción de que la cultura genera un malestar en los seres humanos al suprimir los más intensos deseos de la voluntad de ser desmedidamente libres.

En la segunda parte, la autora nos presenta una exposición sobre la posibilidad de considerar que en Kant existe una teoría sobre la sociabilidad, apoyándose en reglas pragmáticas, el uso de la astucia, el ingenio y el gusto por la conformación de un paradigma estético y político. El primero de estos ensayos, cuarto en el orden del libro, trata sobre la manera en que, para Kant, el conocimiento del ser humano tiene que ser útil, no sólo en cuanto a los fines individuales, sino morales. Un trabajo de interpretación con el cual la Antropología deja de ser un compendio de curiosidades y cobra importancia en cuanto al material que en ella se reúne, con vistas a la orientación de las acciones de los seres humanos en la constitución de sí mismos y de su sociedad. Ahora bien, la filósofa española repara en que el punto de inicio para el estudio de la vida del ser humano, para Kant, está en el tedio, como motor hacia la inquietud y la distracción voluntaria, lo cual origina el resto de la actividad humana en la cultura y la sociedad. Esta búsqueda de salida de la nada lleva al ser humano a la actividad y al encuentro con los otros.

El siguiente ensayo pone en relevancia la importancia que guarda el ingenio dentro de la obra de Kant, como un impulso creativo y productor 
de multiplicidad dentro de la especie humana. Además, juega un papel significativo en la anticipación como ejercicio propio de la libertad del sujeto; en la forma de los talentos del entendimiento, mantiene la búsqueda de leyes y principios racionales en la Naturaleza. De ahí también la necesidad de desarrollar la sagacidad como una habilidad para evitar los errores del conocimiento, así como la destreza en otro tipo de actividades menos regidas por el quehacer de la lógica. La autora analiza los talentos del juicio y los del ingenio los cuales, al combinarse, conforman un talento fundamental del ánimo, al enlazar los conceptos postulados por el ingenio y la dirección del juicio a través de su reflexión. Por último, aborda el concepto de genio como algo invisible, en concordancia con el concepto de espíritu [Geist], cuya peculiaridad radica en la capacidad de invención como manera de anticiparse, al crear nuevas reglas para una nueva época y salir de la cotidianidad, no sólo en el arte, también en la ciencia.

En el último de los ensayos dedicados a la sociabilidad, se aborda el tema de las posibilidades políticas que se desprenden de una sociedad gobernada por el juicio de gusto. En un primer momento, se nos antepone la problemática sobre la transparencia de la realidad frente a nuestros juicios, cuando éstos obedecen a la percepción de lo agradable o desagradable, en la medida en que aquello que puede parecer de una forma, a la postre se revela como todo lo contrario. El ejemplo que la doctora Sánchez Madrid nos refiere de Kant es sobre un hombre que juzga bello un entorno al considerarlo obra de la Naturaleza, pero le repugna como tal si lo descubre como un artificio, aunque, a partir de ello, sea capaz de reconocer su gusto por lo artificial. La autora discute entonces sobre esta paradoja kantiana donde los seres humanos se encuentran al pretender que su comportamiento cumpla con ciertos ideales, mas siempre están revestidos de formas y artificios en la convivencia.

La tercera sección temática versa sobre algunas de las cuestiones que en la actualidad son muy discutidas acerca del pensamiento kantiano: su postura respecto al concepto de raza, el colonialismo y su concepción jurídicopolítica del cosmopolitismo. Aquí, la 
profesora Nuria Sánchez Madrid reconoce la influencia de Onora O'Neill, Reidar Maliks y Katrin Flickschuh. Para comenzar, Sánchez Madrid se aproxima a las dificultades contextuales del pensamiento kantiano a cerca del concepto de raza, para identificar y distinguir aquellos argumentos sobre los cuales Kant se apoya para conservar dicha noción, desde una visión epigenética que obedece a su propensión universalista y antipluralista; mientras que, por otra parte, rechaza las prácticas colonialistas de esclavitud y sometimiento conforme Europa avanza hacia el final del siglo XviII.

En el octavo ensayo, la autora toca la cuestión sobre los límites contextuales históricos que impiden a Kant ampliar el alcance jurídico de su idea de cosmopolitismo, en razón de su propia teoría del Estado, la cual no le permitió asumir una tarea como la de garantizar los recursos materiales para que los individuos participen activamente como ciudadanos; por otra parte, atacó a la aristocracia como obstaculizadora del desarrollo de la sociedad liberal. Respecto a la articulación jurídica de dicho cosmopolitismo, Sánchez Madrid interviene en una discusión que viene desde Paulin Kleingeld, Helga Varden y Alyssa R. Bernstein, acerca de la posibilidad de que un Estado intervenga coactivamente con otro, por medio de las armas, a partir de la conformación de una liga orientada a mantener la paz.

El principal propósito de Sánchez Madrid, en este sentido, es visibilizar las dificultades que esa teoría del Estado presenta, al tratarse de los intereses y el beneficio de los individuos, en aras del cumplimiento de un ideal racional universal; advierte ella que la historicidad irrumpe en el modelo de Kant. Esto nos lleva al tema del último ensayo que compone la obra, donde se revisa la consistencia del estatuto racional del derecho cosmopolita, fundado sobre la concepción de un poder supremo que impone un estatuto moral al que todo el Estado debe someterse, con lo cual, habría que suponer la garantía de un orden similar al de una ley universal, comparable con la gravitación de Newton. La autora se encarga de presentar algunos problemas que enfrenta esta teoría, como la propiedad privada y la desigualdad social, o las posibles derivaciones que el verticalismo jurí- 
dico-político de este modelo conlleva hacia la manutención de un Estado que, en nombre del orden del estatuto racional, se puede convertir en opresor de las individualidades.

Las conclusiones de la obra reflexionan sobre el carácter parcial de sus temas y del pensamiento mismo, para romper con el anquilosado modelo sistemático de la obra kantiana, en donde suele verse como un monumento perfecto y exacto. Por el contrario, lo que la filósofa Nuria Sánchez Madrid se ha propuesto es hacer notar la historicidad de ese pensamiento, en sus intentos por explicar el acontecer de su época y las dificultades que el presente le opone. El Kant de nuestro tiempo, alejado de nuestros problemas, resulta útil y orientador para pensar el presente y la conciencia que de él se ha obtenido con la Ilustración.

Por último, cabe señalar que el libro viene prologado por José Luis Villacańas, académico de la misma entidad y antiguo profesor de la autora, y con un epílogo de Pablo Oyarzun, de la Universidad de Chile: un par de escritos que valoran la pertinencia y dan cuenta de la suficiencia de esta obra. 